\title{
Estudo retrospectivo de ovariossalpingo-histerectomia em cadelas e gatas atendidas em Hospital Veterinário Escola no período de um ano
}

\author{
[Retrospective ovariosalpingohisterectomy study in bitches and queens assisted at a \\ Veterinary School Hospital during one year] \\ C.P. Balthazar da Silveira ${ }^{2}$, E.A.A. Machado ${ }^{1}$, W.M. Silva ${ }^{1}$, T.C.M.S. Marinho ${ }^{1}$, A.R.A. Ferreira ${ }^{1}$, \\ C.P. Bürger ${ }^{2}$, J.M. Costa Neto ${ }^{1}$ \\ ${ }^{1}$ Escola de Medicina Veterinária - Universidade Federal da Bahia - Salvador, BA \\ ${ }^{2}$ Faculdade de Ciências Agrárias e Veterinárias - UNESP - Jaboticabal, SP
}

\begin{abstract}
RESUMO
Realizou-se um estudo retrospectivo das indicações de ovariossalpingo-histerectomia - eletiva e terapêutica - no período de um ano. Foram analisados 193 prontuários de cadelas e gatas atendidas em Hospital Veterinário Escola, entre março de 2010 e março de 2011, levando em consideração a espécie, a idade e o uso ou não de anticoncepcional. Constatou-se que a demanda de OSH com caráter terapêutico $(78,8 \%)$ é consideravelmente mais alta que a eletiva $(21,2 \%)$. Observou-se que as anormalidades reprodutivas detectadas com maior frequência foram piometra $(53,36 \%)$ e complicações obstétricas $(25,38 \%)$ e que o uso de anticoncepcional foi o fator relevante para o delineamento desses quadros.
\end{abstract}

Palavras-chave: ovariossalpingo-histerectomia eletiva e terapêutica, cadela, gata

\begin{abstract}
An ovariosalpingohisterectomy procedure indication - elective and therapeutic - retrospective study was conducted during one year. Record files from 193 bitches and queens assisted at a Veterinary School Hospital between March 2010 and March 2011 were analyzed, considering species, age and contraceptive use. It was possible to conclude that the therapeutic OSH demand (78.8\%) is remarkably superior to the elective OSH request (21.2\%). It was also observed that the most frequently detected reproductive abnormalities were pyometra (53.36\%) and gestational complications (25.38\%), and also that the use of contraceptives is a relevant factor for those events.
\end{abstract}

Keywords: elective and therapeutic ovariosalpingohisterectomy, bitch, queen

\section{INTRODUÇÃO}

A ovariossalpingo-histerectomia $(\mathrm{OSH})$ é um procedimento cirúrgico frequentemente empregado na medicina veterinária, a qual consiste na realização de laparotomia com ablação dos ovários, trompas e útero. Há basicamente três abordagens para a execução de tal procedimento: por videolaparoscopia (Schiochet et al., 2009), pela lateral do abdômen (flanco) (Elices Minguez et al., 2005) e pelo método tradicional (linha média ventral) (Howe, 2006).
Independentemente do acesso, a OSH tem sua importância por ser a técnica utilizada no tratamento de partos patológicos (Luz et al., 2005; Howe, 2006), na prevenção de neoplasias mamárias (Fonseca e Daleck, 2000), no controle populacional de pequenos animais, nos procedimentos de esterilização eletiva (prevenção do cio e/ou de prenhez indesejada) (Howe, 2006) e na prevenção e tratamento das doenças do trato reprodutivo (tumores ovarianos, hiperplasia endometrial cística piometra, torção, prolapso e neoplasias uterinas) (Martins e Lopes, 2005; Mostachio et al., 2008; Pliego et al., 2008; Rolim et al., 2010).

Recebido em 24 de outubro de 2011

Aceito em 6 de setembro de 2012

E-mail: bibiagarvana@gmail.com 
Por consistir em um procedimento simples e de fácil execução, rotineira em clínicas veterinárias privadas e públicas e amplamente utilizada na didática de ensino da técnica cirúrgica em hospitais-escola, o objetivo deste trabalho foi realizar um estudo retrospectivo de ovariossalpingo-histerectomia, em cadelas e gatas atendidas em Hospital Veterinário Escola, no período compreendido entre março de 2010 e março de 2011, levando em consideração os seguintes dados: espécie, idade, uso ou não de anticoncepcional e indicação para realização do procedimento (eletivo ou terapêutico).

\section{MATERIAL E MÉTODOS}

Foram analisadas 193 fichas de cadelas e gatas submetidas ao procedimento de ovariossalpingohisterectomia em Hospital Veterinário Escola, entre março de 2010 e março de 2011. Das fichas clínicas avaliadas foram recolhidos dados relativos à anamnese, ao exame físico, a exames complementares e de imagem radiográficos e ultrassonográficos - e indicação do procedimento. Os dados foram organizados em dois grupos principais: grupo 1, composto por pacientes submetidos ao procedimento cirúrgico de $\mathrm{OSH}$ com caráter eletivo (controle populacional de cães e gatos, prevenção do cio), denominado grupo OSH eletiva, e o grupo 2, formado por pacientes submetidos ao procedimento de OSH por apresentarem alterações patológicas do sistema reprodutor feminino - complicações obstétricas, complexo endometrial cístico/piometra, torção e/ou prolapso uterino, cisto ovariano - integrando o grupo OSH terapêutica.

O grupo OSH terapêutica foi subdivido em outros dois: o primeiro subgrupo, com informações sobre complicações obstétricas, foi subdividido em mais dois grupos menores, de acordo com o procedimento realizado - cesariana com OSH ou somente OSH; no segundo subgrupo, referente a complicações patológicas, estavam os pacientes com diagnóstico de piometra e que foram submetidos à OSH. Em ambos os subgrupos do grupo OSH terapêutica efetuou-se registro de informações sobre o uso ou não de anticoncepcional.

A análise dos dados foi consignada de forma descritiva, e, para testar a frequência dos procedimentos eletivos e terapêuticos, foram feitas análises de qui-quadrado por meio do programa Statistical Package for the Social Sciences, versão 17.0.

\section{RESULTADOS E DISCUSSÃO}

Dos 193 prontuários analisados, 78,8\% pertenciam ao grupo OSH terapêutica, e apenas $21,2 \%$ ao grupo OSH eletiva. A casuística de OSH terapêutica em cadelas foi três vezes maior do que a eletiva $\left(\mathrm{x}^{2}=29,160 ;\right.$ g.l. $=1 ; \mathrm{P}<0,001$; Fig. 1). Em gatas, a casuística de $\mathrm{OSH}$ terapêutica foi cinco vezes maior que a eletiva $\left(\mathrm{x}^{2}=14,235 ;\right.$ g.1.=1; $\mathrm{P}<0,001$; Fig. 1).

Inoe et al. (2004), em estudo retrospectivo de ovariossalpingo-histerectomia, revelaram que os procedimentos de caráter eletivo foram mais frequentes que os terapêuticos, resultado diferindo do observado no presente trabalho. Observou-se que a OSH eletiva não é o procedimento de maior demanda na instituição estudada, sendo nesse aspecto superada pela técnica realizada em caráter terapêutico.

No grupo OSH terapêutica, em seu primeiro subgrupo, referente a complicações obstétricas 30 cadelas e 19 gatas, com idade média de quatro anos -, $27(55,1 \%)$ foram submetidas à cesariana seguida de OSH, enquanto 22 $(44,9 \%)$ somente ao procedimento de OSH. Os pacientes submetidos ao procedimento de cesariana seguida de OSH apresentavam viabilidade fetal pré-cirúrgica e não fizeram uso de anticoncepcional, experimentando um desencadeamento anormal do parto.

Neste estudo, só havia registro de cesariana radical - aquela em que se realiza a OSH após a remoção dos filhotes do útero. A indicação desse procedimento pode ser atribuída principalmente à constatação de inviabilidade uterina, assim como, entre outras razões, ao controle populacional. Sobre este último, pode-se afirmar que é usual, na comunidade atendida pelo Hospital Veterinário Escola, a opção pela esterilização dos pacientes, visto que é incomum entre aquele público a criação com finalidade reprodutiva (o que se deve ao baixo valor de mercado dos animais por eles criados). Por ocasião da cesariana, diversos proprietários solicitam OSH, visando evitar os transtornos decorrentes do cio e de gestações não programadas. 


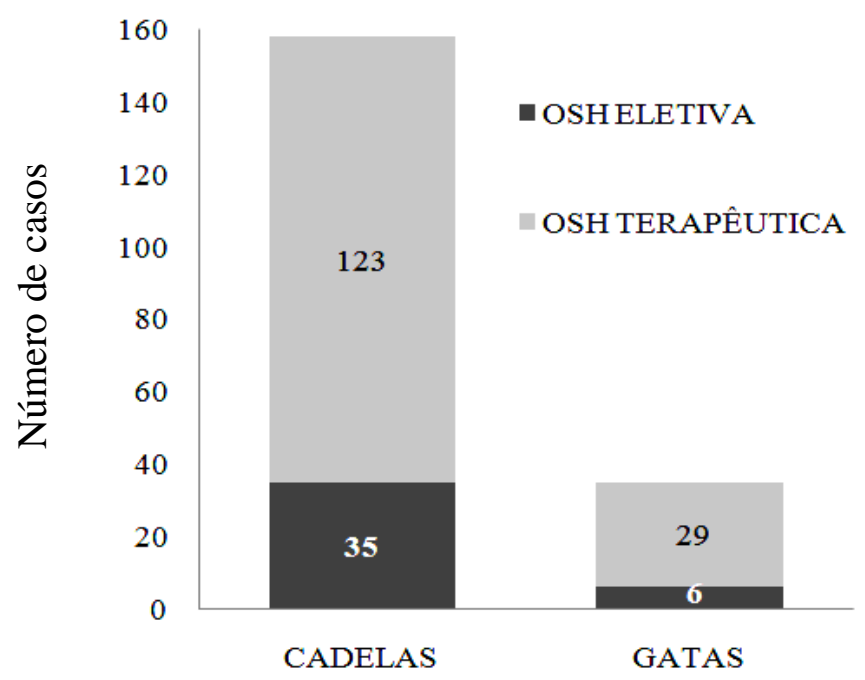

Figura 1. Número de procedimentos de OSH eletiva e terapêutica, em cadelas e gatas.

Os animais submetidos somente à OSH apresentaram inviabilidade/maceração fetal, o que resultou de complicações graves como parto anormal $(81,8 \%)$, prolapso uterino $(9,1 \%)$, torção uterina, $(4,5 \%)$ e trauma em útero gravídico $(4,5 \%)$ (Fig. 2). Destes 22 indivíduos, 11 fizeram o uso de anticoncepcional na gestação, todos apresentando parto anormal, sendo $36,4 \%$ cadelas e $63,6 \%$ gatas; os demais não fizeram uso de anticoncepcional $(\mathrm{n}=11)$, estando entre eles animais com torção uterina, prolapso uterino e trauma, sendo $81,8 \%$ cadelas e $18,2 \%$ gatas.

Luz et al. (2005) citaram fatores maternos e fetais que podem contribuir para distocia. Mencionaram também o uso indiscriminado de progestágenos injetáveis como causa apta a ocasionar distocia. $\mathrm{O}$ acetato de medroxiprogesterona, amplamente utilizado para prevenir a ocorrência do estro em cadelas e gatas (Moreira et al., 2008; Loretti et al., 2004), é contraindicado em pacientes gestantes; quando usado de maneira errônea, a inviabilidade/maceração fetal torna-se um achado comum (González-Domínguez e Maldonado-Estrada, 2006). Evidência prática disso encontra-se no fato de que os 11 pacientes que fizeram o uso de anticoncepcional apresentaram inviabilidade/maceração fetal, já que as altas concentrações de progesterona, de origem exógena, não permitem que ocorra contração uterina, dilatação cervical e expulsão fetal; prolonga-se, assim, a gestação, o que acarreta morte do feto (Luz et al., 2005; Prestes e Landim-Alvarenga, 2006). A baixa casuística de torção e prolapso uterino é compatível com os dados encontrados na literatura (Biddle e Macintire, 2000; Ridyard et al., 2000; Mostachio et al., 2008; Raut et al., 2008).

O segundo subgrupo $(\mathrm{n}=103)$ refere-se aos animais que tiveram confirmação diagnóstica de piometra por meio de exames físicos, complementares e/ou por imagem. A média de idade foi de sete anos, corroborando os dados encontrados em outros estudos, que relataram que a incidência da doença é comum na faixa etária acima de cinco anos (Kim e Kim, 2005; Faria e Norsworthy, 2008; Evangelista et al., 2010), e raros foram os casos em que tal situação ocorreu em animais com menos de um ano de vida (Fan Chen et al., 2007; Moreira et al., 2008).

Neste grupo, detectou-se maior incidência de piometra em cadelas $(90,3 \%)$ do que em gatas $(9,7 \%)$, confirmando o que indica a literatura (Silveira et al., 2007). Isso pode ser explicado pela diferença existente entre o ciclo estral das espécies em questão: as gatas só ovulam quando são estimuladas por meio da cópula, sofrendo menor efeito da progesterona ao longo da vida quando comparadas às cadelas, que apresentam uma fase luteínica extensa (Smith, 2006; Toniollo et al., 2000). 


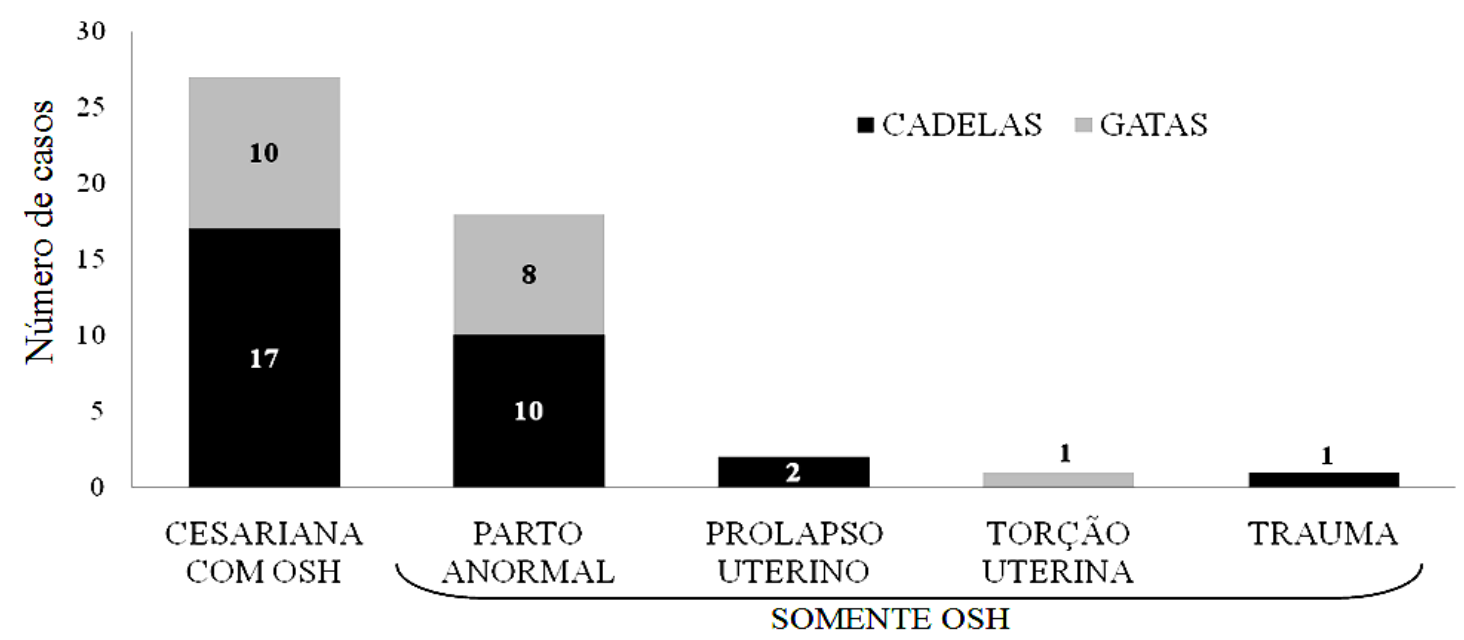

Figura 2. Complicações obstétricas de cadelas e gatas relacionadas ao tipo de procedimento realizado.

A piometra aberta $(84,4 \%)$ foi mais frequente que a fechada (15,5\%) nas duas espécies (Fig. 3). A maior incidência da piometra aberta foi compatível com os dados descritos na literatura (Hedlund et al., 2008; Siqueira et al., 2008). Carreira e Pires (2005) e Weiss et al. (2004) descreveram que o desenvolvimento da piometra é precedido pela hiperplasia endometrial cística, a qual consiste em uma resposta exagerada e anormal do endométrio à estimulação da progesterona crônica e repetidamente, levando ao acúmulo de fluido no lúmen uterino e glândulas endometriais; quando invadidas por bactérias oportunistas como Escherischia coli (Smith, 2006; Siqueira et al., 2008; Lara et al., 2008; Pretzer, 2008), pode-se estabelecer infecção em seu interior.

Neste estudo, cinco cadelas $(5,4 \%)$ fizeram uso de anticoncepcional e apresentaram piometra (duas abertas e três fechadas) e, dentre as gatas, três $(30 \%)$ fizeram uso de anticoncepcional e apresentaram piometra aberta. Essa doença tem sido descrita em animais que fizeram uso de agentes contraceptivos, como os análogos da progesterona (acetato de medroxiprogesterona), cuja administração prolongada e indiscriminada pode dar ensejo a complicações intrauterinas importantes, dados que guardam consonância com os achados deste estudo (Kim e Kim, 2005; Moreira et al., 2008; Keskin et al., 2009). Frequentemente se observa o aparecimento de hiperplasia endometrial cística/piometra ou mesmo complicações obstétricas em pacientes que já fizeram algum uso de contraceptivo (Kim e Kim, 2005; González-Domínguez e
Maldonado-Estrada, 2006; Moreira et al., 2008; Keskin et al., 2009). Situações como as descritas anteriormente podem tornar necessária a realização de OSH de caráter emergencial (Pretzer, 2008).

Os sinais clínicos mais frequentes para as duas formas clínicas foram: secreção vaginal mucopurulenta a serossanguinolenta (piometra aberta), abdômen distendido, apatia, letargia, desidratação, depressão, êmese, anorexia, polidipsia e poliúria. Os animais com piometra fechada apresentaram também sinais de toxemia e septicemia e necessitaram de intervenção cirúrgica em caráter emergencial, sinais clínicos estes também descritos por Kaymaz et al. (1999), Moreira et al. (2008), Pretzer (2008), Faria e Norsworthy (2008) e Evangelista et al.(2010), nos casos de piometra aberta e fechada.

$\mathrm{Na}$ rotina cirúrgica adotada na instituição em questão, os protocolos pré e pós-operatórios para piometra diferem em seu caráter, a depender dos sinais clínicos do paciente. Animais com quadros agudos de piometra que apresentam sinais clínicos discretos são primeiramente avaliados, submetidos a análises laboratoriais e de imagem, estabilizados e só então encaminhados para a realização de procedimento cirúrgico. Indivíduos portadores de quadros mais graves, por sua vez, necessitam de intervenção emergencial, com protocolo distinto: são inicialmente estabilizados e logo submetidos à cirurgia, não se aguardando o resultado final de todos os exames préoperatórios para que se dê início à intervenção. 


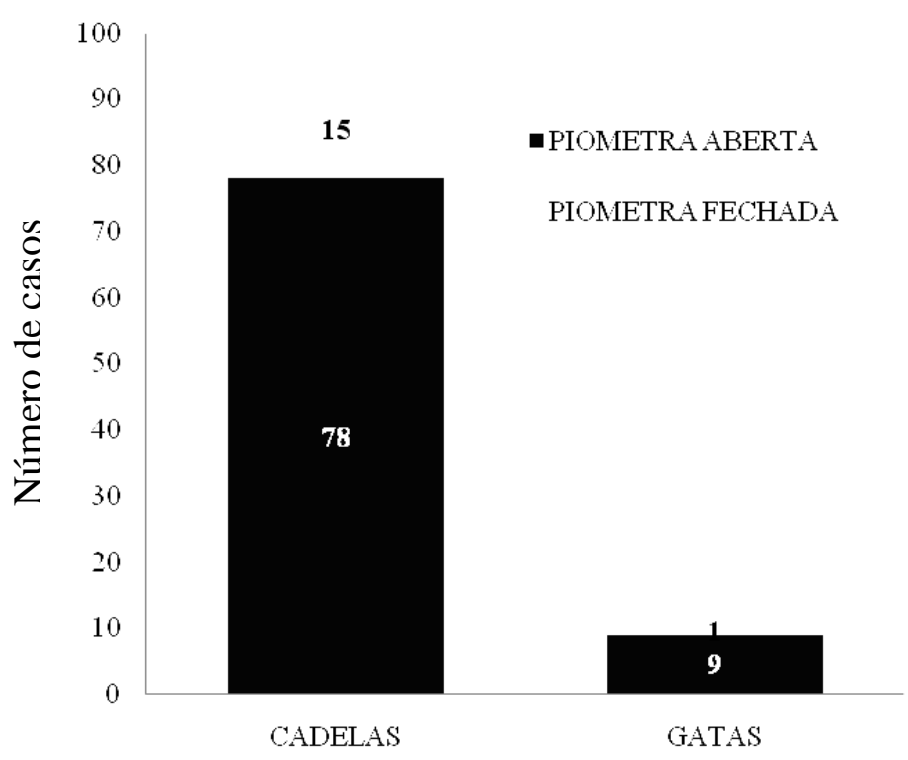

Figura 3. Ocorrência de piometra aberta e fechada entre cadelas e gatas.

\section{CONCLUSÕES}

As ovariossalpingo-histerectomias com intuito terapêutico foram mais frequentes do que as eletivas, especialmente no subgrupo referente à piometra, muito embora o grupo relativo a complicações obstétricas tenha tido um papel significativo. Vislumbra-se, assim, a necessidade de maior divulgação de informações sobre a importância dos procedimentos de castração de fêmeas, tomando como público-alvo a comunidade que se beneficia dos serviços prestados pelos Hospitais Veterinários Escola. Tal medida tem como escopo a redução das taxas de OSH terapêuticas e, a partir disso, a melhoria na qualidade de vida dos animais e de seus proprietários.

\section{REFERÊNCIAS}

AMAKU, M.; DIAS, R.A.; FERREIRA, F. Rev. Panam. Salud Publica, v.25, p.300-304, 2009.

BIDDLE, D.M.; MACINTIRE, D.K. Obstetrical emergencies. Clin. Tech. Small Anim. Pract., v.15, p.88-93, 2000.

CARREIRA, P.R.; PIRES, M.A. Hiperplasia quística do endométrio em cadelas. RPCV, v.100, p.5-16, 2005.

ELICES MINGUEZ, R.; MARTINEZ-DARVE, J.G.; MORÁN CUESTA, M. Ovariohisterectomia de gatas e cadelas pelo flanco. Rev. Bras. Reprod. Anim., v.29, p.151-158, 2005.
EVANGELISTA, L.S.M.; QUESSADA, A.M.; ALVES, R.P.A. et al. Função renal de cadelas com piometra antes e após ovariosalpingohisterectomia. Acta Vet. Bras., v.4, p.153-161, 2010.

FAN CHEN, R.F.; ADDEO, P.M.D.; SASAKI, A.Y. Piometra aberta em uma cadela de 10 meses. Rev. Acad. Agrar. Ambient., v.5, p.317-322, 2007.

FARIA, V.P.; NORSWORTHY, G.D. Case reportPyometra in a 13-year-old neutered queen. JFMS, v.10, p.185-187, 2008.

FONSECA, C.S.; DALECK, C.R. Neoplasias mamárias em cadelas: influência hormonal e efeitos da ovário-histerectomia como terapia adjuvante. Cienc. Rural, v.30, p.731-735, 2000.

GONZÁLEZ-DOMÍNGUEZ, M.S.; MALDONADOESTRADA, J.G. Gestación prolongada asociada con la prescripción inadecuada de medroxiprogesterona acetato. ¿Es racional y ético el uso de progestágenos exógenos en perras?. RCCP, v.19, p.442-450, 2006.

HEDLUND, C.S. Cirurgia dos sistemas reprodutivos e genital. In: FOSSUM, T.W. (Ed.). Cirurgia de pequenos animais. 3. ed. São Paulo: Roca, 2008. Cap.26, p.702-774.

HOWE, L.M. Surgical methods of contraception and sterilization. Theriogenology, v.66, p.500-509, 2006.

INOE, A.P.; ZAFANELI, M.C.G.; CUNHA, C.G. et al. Estudo retrospectivo de 228 ovariosalpingohisterectomias realizadas no Hospital veterinário da UNIPAR no período de janeiro de 1999 a julho de 2004. Arq. Cienc. Vet. Zool. Unipar, v.7, p.28, 2004. 
KAYMAZ, M.; BASTAN, A.; ERÜNAL, N. et al. The use of laboratory findings in the diagnosis of CEH-pyometra complex in the bitch. Turk. J. Vet. Anim. Sci., v.23, p.127-133, 1999.

KESKIN, A.; YILMAZBAS, G.; YILMAZ, R. et al. Pathological abnormalities after long-term administration of medroxyprogesterone acetate in a queen. JFMS, v.11, p.518-521, 2009.

KIM, K.S.; KIM, O. Cystic endometrial hyperplasia and endometritis in a dog following prolonged treatment of medroxyprogesterone acetate. J. Vet. Sci., v.6, p.81-82, 2005.

LARA, V.M.; DONADELI, M.P.; CRUZ, F.S.F.; CARREGARO, A.B.; Multirresistência antimicrobiana em cepas de Escherichia coli isoladas de cadelas com piometra. Arq. Bras. Med. Vet. Zootec., v.60, p.1032-1034, 2008.

LORETTI, A.P.; ILHA, M.R.S.; BREITSAMETER, I.; FARACO, C.S. Clinical and pathological study of feline mammary fibroadenomatous change associated with depot medroxyprogesterone acetate therapy. Arq. Bras. Med. Vet. Zootec., v.25, p.270-274, 2004.

LUZ, M.R.; FREITAS, P.M.C; PEREIRA, E.Z. Gestação e parto em cadelas: fisiologia, diagnóstico de gestação e tratamento das distocias. Rev. Bras. Reprod. Anim., v.29, p.142-150, 2005.

MARTINS, L.R; LOPES, M.D. Pseudociese canina. Rev. Bras. Reprod. Anim., v.29, p.137-141, 2005.

MOREIRA, H.R.; MIRANDA, S.A.; BRITO, A.B. $e t$ al. Complexo hiperplasia endometrial cística-piometra em uma cadela tratada com acetato de medroxiprogesterona como método contraceptivo. Rev. Port. Ciênc. Vet., v.103, p.233-238, 2008.

MOSTACHIO, G.Q.; VICENTE, W.R.R.; CARDILLI, D.J. et al. Prolapso uterino em gata e retroflexão uterina em cadela. $C A B$, v.9, p.801-805, 2008.

PLIEGO, C.M.; FERREIRA, M.L.G.; FERREIRA, A.M.R. Leiomiossarcoma uterino em cadela. Vet. Zootec.,v.15, p.40-41, 2008.

PRESTES, N.C.; LANDIM-ALVARENGA, F.C. Possibilidades auxiliares para intervir no parto distócico. In: __. Obstetrícia Veterinária. Rio de janeiro: Guanabara Koogan, 2006. Cap.14, p.204-219.
PRETZER, S.D. Clinical presentation of canine pyometra and mucometra: a review. Theriogenology, v.70, p.359-363, 2008.

RAUT, B.M.; RAGHUWANSHI, D.S.; UPADHYE, S.V. et al. Uterine Torsion in a Bitch. Vet. World, v.1, p.212, 2008.

RIDYARD, A.E.; WELSH, E.A.; GUNN-MOORE, D.A. Successful treatment of uterine torsion in a cat with severe metabolic and haemostatic complications. JFMS, v.2, p.115-119, 2000.

ROLIM, V.M.; PINTO, T.M.; LOPES, L.M.A. et al. Disgerminoma bilateral e hiperplasia endometrial cística com piometra em cadela. Acta Sci.Vet., v.38, p.337-340, 2010.

SCHIOCHET, F.; BECK, C.A.C.; SILVA, A.P.F.F. et al. Ovário-histerectomia laparoscópica em felinos hígidos: estudo comparativo de três métodos de hemostasia. Arq. Bras. Med. Vet. Zootec., v.61, p.369377, 2009.

SMITH, F.O. Canine pyometra. Theriogenology, v.66, p.610-612, 2006.

SILVEIRA, D.S.; BASSI, P.B.; OTERO, L.B. et al. Piometra em caninos e felinos: perfil leucocitário, prevalência nas espécies e sinais clínicos. IN: CONGRESSO DE INICIAÇÃO CIENTÍFICA PESQUISA E RESPONSABILIDADE AMBIENTAL, 16., 2007, Pelotas. Online. Disponível em: <http://www.ufpel.edu.br/cic/2007/cd/ index.php> Acessado em: 21 mai. 2011. (Resumo).

SIQUEIRA, A.K.; RIBEIRO, M.G.; SALERNO, T. et al. Perfil de sensibilidade e multirresistência em linhagens de Escherichia coli isoladas de infecção do trato urinário, de piometra e de fezes de cães. Arq. Bras. Med. Vet. Zootec., v.60, p.1263-1266, 2008.

TONIOLlO, G.H.; FARIA Jr, D.; LEGA, E. et al. Piometra na espécie felina - Relato de um caso em Panthera onca. Braz. J. Vet. Res. Anim. Sci., v.37, n.2, 2000.

VOITH, V.L. The impact of companion animal problems on society and the role of veterinarians. Vet. Clin. N. Am. - Small., v.39, p.327-345, 2009.

WEISS, R.R.; CALOMENO, M.A.; SOUSA, R.S. et al. Avaliação histopatológica, hormonal, e bacteriológica da piometra na cadela. Arch. Vet. Sci., v.9, p.81-87, 2004. 